\title{
Genetics of larval urea and ammonia tolerance and cross-tolerance in Drosophila melanogaster
}

\author{
DANIEL J. BORASH \& MASAKAZU SHIMADA* \\ Department of Systems Sciences - Biology, University of Tokyo (Komaba Campus), Meguro-ku, \\ Tokyo, Japan 153-8902
}

\begin{abstract}
Five laboratory populations of Drosophila melanogaster previously selected for over 60 generations for larval resistance to ammonium chloride $\left(\mathrm{NH}_{4} \mathrm{Cl}\right)$, and five populations selected for over 60 generations for larval resistance to urea, were investigated to determine the genetic mechanisms through which such tolerance had evolved. To examine the genetics of tolerance to urea and ammonia, egg-to-adult survivorship and developmental time were measured at two different $\mathrm{NH}_{4} \mathrm{Cl}$ levels and two different urea levels for each selection regime relative to the control lines, and among reciprocal crosses between each selection regime and the control lines. To examine tolerance to novel nitrogenous compounds (cross-tolerance), egg-to-adult survivorship and developmental time were measured at two different $\mathrm{NH}_{4} \mathrm{Cl}$ levels and two different urea levels for reciprocal crosses between the selection lines. Dominance is a major genetic factor in egg-to-adult survivorship in the presence of either urea or ammonia, while cross-tolerance to novel nitrogenous compounds also shows dominance as a major genetic mechanism controlling egg-to-adult survivorship. Dominance and $\mathrm{X}$-linkage appear to be factors affecting developmental time in the presence of either urea or ammonia, although we could not exclude cytoplasmic inheritance as influencing our results. Crosstolerance to novel nitrogenous compounds shows dominance and $\mathrm{X}$-linkage as the main genetic factors controlling developmental time. We develop a simple hypothesis, in accordance with the results, that there may be two X-linked loci: one controlling urea tolerance and one controlling ammonia tolerance, and one autosomal locus exerting a pleiotropic control of tolerance. However, many other possibilities exist.
\end{abstract}

Keywords: ammonia tolerance, density-dependent natural selection, dominance, stress resistance, urea tolerance, X-linkage.

\section{Introduction}

Evolutionary ecology seeks to explain the mechanisms by which life history characteristics may be moulded by the environment. Changes in a population's density have been shown to have profound effects on the environment (Hageman et al., 1990; Borash et al., 1998), and such temporal environmental changes can affect the direction and magnitude of selection acting upon the population itself. Density-dependent natural selection has been demonstrated to affect a wide range of physiological and behavioural characters in Drosophila melanogaster (Burnett et al., 1977; Botella et al., 1985; Joshi \& Mueller, 1988; Mueller, 1995). In a population

*Correspondence. E-mail: mshimada@balmer.c.u-tokyo.ac.jp under density regulation, the build up of metabolic waste products and the limitation of resources (food and space) comprise two powerful factors affecting the evolution of life history characteristics (Borash et al., 1998).

Five populations of Drosophila melanogaster selected for larval tolerance to extreme levels of urea (Shiotsugu et al., 1997) were assayed in order to determine the underlying genetics controlling their urea tolerance. Joshi et al. (1996) found that hybrid populations demonstrated higher egg-to-adult survivorship and faster developmental times than their midparent values, indicating that dominance was a major factor governing these traits in the urea-selected populations. They also found a significant X-linkage effect controlling tolerance to high levels of urea (Joshi et al., 1996). 
Borash et al. (1998) reported that ammonia was the primary metabolic waste product of Drosophila melanogaster larvae. New populations were initiated, which were selected for tolerance to ammonium chloride, along with selection to create a new series of urea-tolerant populations. As expected, both ammoniaselected populations and urea-selected populations became increasingly more tolerant to their respective selection regime (Borash et al., 2000a; Borash et al., 2000b). However, populations selected for tolerance to extreme levels of ammonia demonstrated significant cross-tolerance to urea for egg-to-adult survivorship and developmental time. Urea-selected populations also displayed cross-tolerance to ammonia for the same traits (Borash et al., 2000a). Urea and ammonia are compounds with different cytotoxic effects on the organism (Somero \& Yancey, 1997; Borash et al., 1998). Hoffmann \& Parsons (1991) have also recognized many cases in which cross-tolerance to different stresses arise. Stresses have a wide range of effects on the organism, both biochemical and physiological, and, if mechanisms of resistance are pleiotropic, selection for one toxin may impart partial, if not complete, tolerance to other compounds.

Here, we detail a series of experiments, investigating whether outbred, replicate, laboratory populations of Drosophila melanogaster which have been selected for ammonia-tolerance, demonstrate similar genetic (dominance and X-linkage) control of egg-to-adult survivorship and developmental time, as was seen in urea-selected populations (Joshi et al., 1996). Finally, we seek to determine whether the observed cross-tolerance, ammonia-selected population's ability to tolerate urea and the urea-selected population's ability to tolerate ammonia, shows dominance and/or $\mathrm{X}$-linkage.

\section{Materials and methods}

Five ammonia-selected, five urea-selected, and five unselected control populations were derived in autumn 1996, from the five Mueller UU populations (Joshi \& Mueller, 1996). The UU populations $\left(\mathrm{UU}_{1}, \ldots, \mathrm{UU}_{5}\right)$ were themselves derived from the Rose B populations (Rose, 1984). All UU populations were maintained on banana-molasses medium, 24:0 light:dark, $25^{\circ} \mathrm{C}$, uncontrolled humidity, and had a generation time of $\approx 3$ weeks. The UU populations were kept uncrowded (60-80 eggs per 8-dram shell vial) as larvae, with eclosing adults being kept at a low adult density of $\approx 60$ adults per 8 -dram vial, and transferred to fresh food three times a week.

The five AX (ammonia-selected) populations were created in 1996, with 60 vials inoculated with $60-80$ eggs each. Prior to egg collection, ammonium chloride $(\mathrm{pH} \approx 5.5$, equivalent to the standard $\mathrm{pH}$ of the food medium) was added to the food before it was dispensed into vials. After egg collection, plastic sleeves were inserted into vials, so pupae could be removed before adult emergence. After a majority $(>90 \%)$ of the larvae had pupated, sleeves were removed and placed into plexiglass or plastic cages with a standard banana-molasses food plate. This procedure ensures that only larvae would be exposed to the food containing ammonia (Borash et al., 2000a). When a large proportion of larvae $(>50 \%)$ from all replicate populations were observed to be surviving to adulthood, the level of ammonia was increased in the ensuing generation. The UX selection regime was maintained in a similar manner, with the exception that urea $(\mathrm{pH} \approx 5.5)$ was added to the food medium before being dispensed into vials. The AUC (AmmoniaUrea-Control) regime served as a control; larvae were not exposed to excessive levels of either ammonia or urea.

Before any experiments were performed, all populations were removed from selection for two generations and reared in identical larval and adult conditions, to ensure the removal of any environmental and maternal effects, which may confound the observance of genetic differentiation among the selection regimes. Assays were performed after 63 generations of selection for replicate populations 1, 2, and 3 of each selection regime, and after 64 generations of selection for replicate populations 4 and 5 of each selection regime.

\section{Hybrid preparation}

Sixty vials with approximately $60-80$ eggs per vial were collected from populations of each selection regime bearing the same subscripted indices (i.e. $\mathrm{AX}_{1}, \mathrm{UX}_{1}$, and $\left.\mathrm{AUC}_{1}\right)$. Eclosing adults were removed from vials, every $8 \mathrm{~h}$, using $\mathrm{CO}_{2}$ anaesthesia. Males and females were sequestered in separate vials. Vials were periodically checked for the presence of larvae, indicating that at least one female in that particular vial was not a virgin, and if a vial contained any larvae, it was eliminated from the experiment.

From each replicate block (AX $i, \mathrm{UX} i, \mathrm{AUC} i: i=1-5)$ nine populations were created using the virgin adults collected. This consisted of a complete reciprocal cross among each block, resulting in the creation of six hybrid populations (e.g. AUC female $\times \mathrm{AX}$ male) and three parental populations (e.g. AUC female $\times$ AUC male). A minimum of 350 flies of each sex, comprising each cross, were dumped into cages and given a standard banana-molasses food plate with a liberal amount of yeast paste on top to stimulate female oviposition. After 
$48 \mathrm{~h}$, plates were replaced and flies were allowed to lay eggs $\left(F_{1}\right)$ over an 8 -h period.

\section{Developmental time and viability}

From each of the nine populations, exactly $60 F_{1}$ eggs were collected on pieces of agar that was non-nutritive to ensure that it would not affect the concentration of the nitrogenous compound added to the food medium. Eggs were placed into vials containing one of five food treatments (plain food, $0.225 \mathrm{~mol}$ urea, $0.450 \mathrm{~mol}$ urea, $0.50 \mathrm{~mol} \mathrm{NH}_{4} \mathrm{Cl}$, or $1.0 \mathrm{~mol} \mathrm{NH}_{4} \mathrm{Cl}$ ). Five replicate vials per food treatment for each of the nine $F_{1}$ populations were created. All vials were housed in an incubator room $\left(25^{\circ} \mathrm{C}, 24: 0\right.$ light:dark, and uncontrolled humidity) throughout the experiment.

Previous experiments did not show parental line differences in developmental time when reared on plain food (Borash et al., 2000a); checks for eclosion were performed every $12 \mathrm{~h}$. Emerging adults were removed, using $\mathrm{CO}_{2}$ anaesthesia, and the time and gender of each fly were recorded. Checks continued, being discontinued only after a period of $48 \mathrm{~h}$ had elapsed in which no flies had emerged.

\section{Statistics}

All data were analysed using statistica version 5 (StatSoft, Release 5.0, 1995, Hamburg, FRG). For eggto-adult survivorship, population and food type were treated as fixed effects, whereas replicate (block) number was set as a random effect. Viability data were arc-sin transformed prior to analysis, in order to normalize the data; however, as transformed data yielded the same results as the non-transformed data, we present the analysis of the non-transformed data. Multiple comparisons were performed using the Tukey-Kramer method (Freeman \& Tukey, 1950). For developmental time, population, food type, and sex of emergent adult were set as fixed effects, whereas replicate (block) was set as a random effect. In order to determine whether there was a significant vial effect on developmental time, the ANOVA incorporated two nested components, a population $\times$ block $\times$ food interaction nested within vial, and a population $\times$ block $\times$ food interaction nested within vial $\times$ sex. A separate ANOVA was performed on $F_{1}$ hybrids and the mid-parents comprising the hybrid populations, to ascertain whether dominance could be observed. Additionally, an ANOVA was performed between the $F_{1}$ hybrids to determine the degree of $\mathrm{X}$-linkage. The statistical methods used were the same as those used by Joshi et al. (1996).

\section{Results}

\section{Egg-to-adult survivorship}

Urea and ammonia tolerance. The ANOvA revealed no differences in egg-to-adult survivorship between purebred and hybrid populations when they were reared as larvae on food containing no urea (Fig. 1A). Food containing low levels of urea $(0.225 \mathrm{~mol})$ significantly reduced egg-to-adult survivorship in all populations, except for the UX pure-bred populations. Food containing high levels of urea $(0.45 \mathrm{~mol})$ reduced egg-to-adult survivorship to a greater extent, in all populations. The ANOVA for dominance revealed a significant population $\times$ food interaction $\left(F_{8,32}=3.47\right.$, $P<0.01$ ), thus permitting multiple comparisons to be made. On food containing low or high levels of urea, both hybrid lines (AUC female $\times$ UX male and UX female $\times$ AUC male) showed significantly greater survivorship relative to the mid-parent value of the UX and AUC pure-bred lines. The ANOVA for X-linkage revealed a significant population $\times$ food interaction $\left(F_{8,32}=3.08\right.$, $P<0.025)$, thus permitting multiple comparisons to be made. The UX female $\times$ AUC male lines displayed greater survivorship than the AUC female $\times$ UX male lines, on both low and high urea levels. As the UX female $\times$ AUC male $F_{1}$ male hybrids inherit their $X$ chromosome from the mother, this is an indication of $\mathrm{X}$-linkage or of cytoplasmic inheritance affecting urea tolerance. Even though we did not conduct assays to discriminate between these two effects, for the sake of brevity, we will treat the effect as one of X-linkage. Ideally, measuring X-linkage would be best performed using the sex ratio of emergent adults, however, we cannot be sure that an equal number of male and female eggs were placed into each vial at the start of the experiment.

There were no differences seen between pure-bred and hybrid populations when they were reared as larvae on food containing no ammonia (Fig. 1B). Food containing low levels of ammonia $(0.50 \mathrm{~mol})$ significantly reduced egg-to-adult survivorship in all populations, except for the AX pure-bred populations. High levels of ammonia $(1.00 \mathrm{~mol})$ reduced egg-to-adult survivorship to a greater extent, in all populations. On food containing low or high levels of $\mathrm{NH}_{4} \mathrm{Cl}$, both hybrid lines (AUC female $\times$ AX male and AX female $\times$ AUC male) showed enhanced survivorship relative to the midparent value of the AX and AUC pure-bred lines. The AX female $\times$ AUC male lines displayed greater survivorship than the AUC female $\times$ AX male lines, on both low and high ammonia levels, indicating that $\mathrm{X}$-linkage may also play a role in aiding egg-to-adult survivorship in the nitrogenous-compound-tolerant populations. 


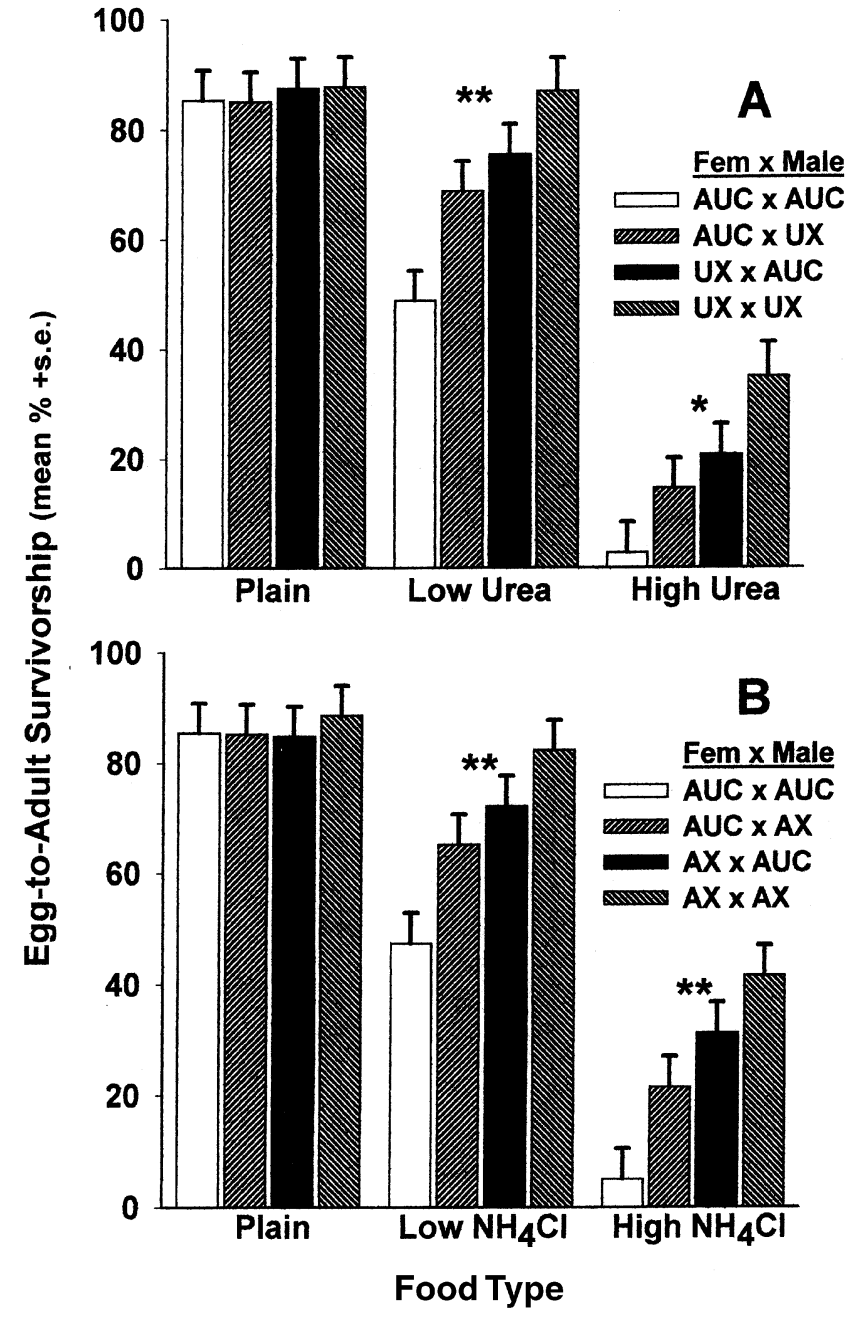

Fig. 1 Effect of larval exposure to urea and ammonia on eggto-adult survivorship in Drosophila melanogaster. (A) Urea. All populations were measured on three types of food: plain banana food, banana food supplemented with $0.225 \mathrm{~mol}$ urea (Low), and banana food supplemented with $0.45 \mathrm{~mol}$ urea (High). (B) Ammonia. All populations were measured on three types of food: plain banana food, banana food supplemented with $0.50 \mathrm{~mol} \mathrm{NH}_{4} \mathrm{Cl}$ (Low), and banana food supplemented with $1.00 \mathrm{~mol} \mathrm{NH}_{4} \mathrm{Cl}$ (High). The error bars are standard errors around the mean of the five replicate populations. Asterisks represent the significance of dominance controlling tolerance to the specific nitrogenous compound. Dominance is revealed by a greater proportion of both hybrid populations surviving to adulthood, relative to their mid-parents. $* P<0.05 ; * * P<0.01$.

Cross-tolerance. When reared as larvae on plain food, $\mathrm{AX}, \mathrm{UX}$, and their reciprocal hybrids were equal in eggto-adult survivorship (Fig. 2). Low levels of urea $(0.225 \mathrm{~mol})$ reduced egg-to-adult survivorship in all populations, except for the UX pure-bred, however, all populations' viabilities declined when reared under high

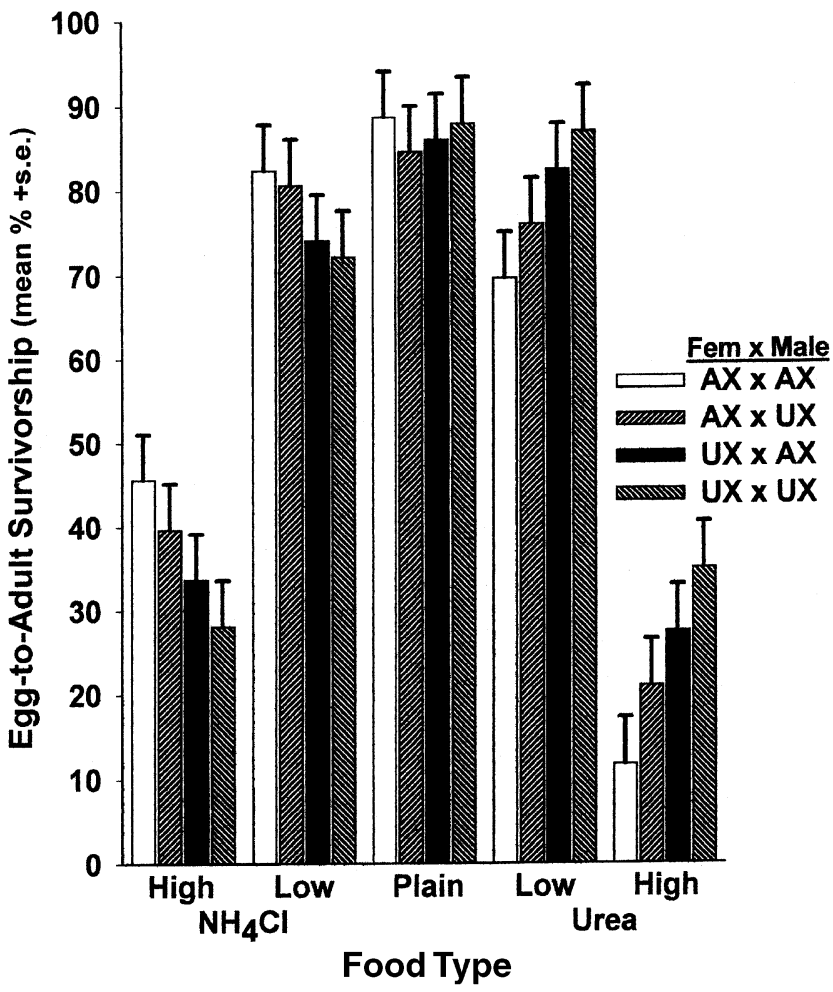

Fig. 2 Effect of larval exposure to urea and ammonia on eggto-adult survivorship and cross-tolerance in Drosophila melanogaster. All AX, UX, and their hybrid populations were measured on five types of food: plain banana food, banana food with $0.225 \mathrm{~mol}$ urea (Low), banana food with $0.45 \mathrm{~mol}$ urea (High), banana food with $0.50 \mathrm{~mol} \mathrm{NH}_{4} \mathrm{Cl}$ (Low), and banana food with $1.00 \mathrm{~mol} \mathrm{NH}_{4} \mathrm{Cl}$ (High). The error bars are standard errors around the mean of the five replicate populations.

urea conditions. Exposure to low levels of $\mathrm{NH}_{4} \mathrm{Cl}$ $(0.50 \mathrm{~mol})$ reduced egg-to-adult survivorship in all populations, except for the AX pure-bred. However, all populations' viabilities declined when reared under high ammonia conditions. UX populations displayed significantly $(P<0.05)$ superior egg-to-adult survivorship on either low or high levels of $\mathrm{NH}_{4} \mathrm{Cl}$, relative to the AUC control populations on the same food (Fig. 3). The AX populations displayed superior egg-to-adult survivorship on either low or high levels of urea, relative to the AUC control populations on the same food type (Fig. 3).

Hybrid populations (AX female $\times$ UX male and $U X$ female $\times$ AX male) did not display superior egg-to-adult survivorship relative to the mid-parent value, indicating that dominance does not significantly affect survivorship for nitrogenous-compound-tolerant populations in novel environments (Fig. 2). As the AX female $\times$ UX male populations showed enhanced survivorship on food containing low or high levels of $\mathrm{NH}_{4} \mathrm{Cl}$, relative to 


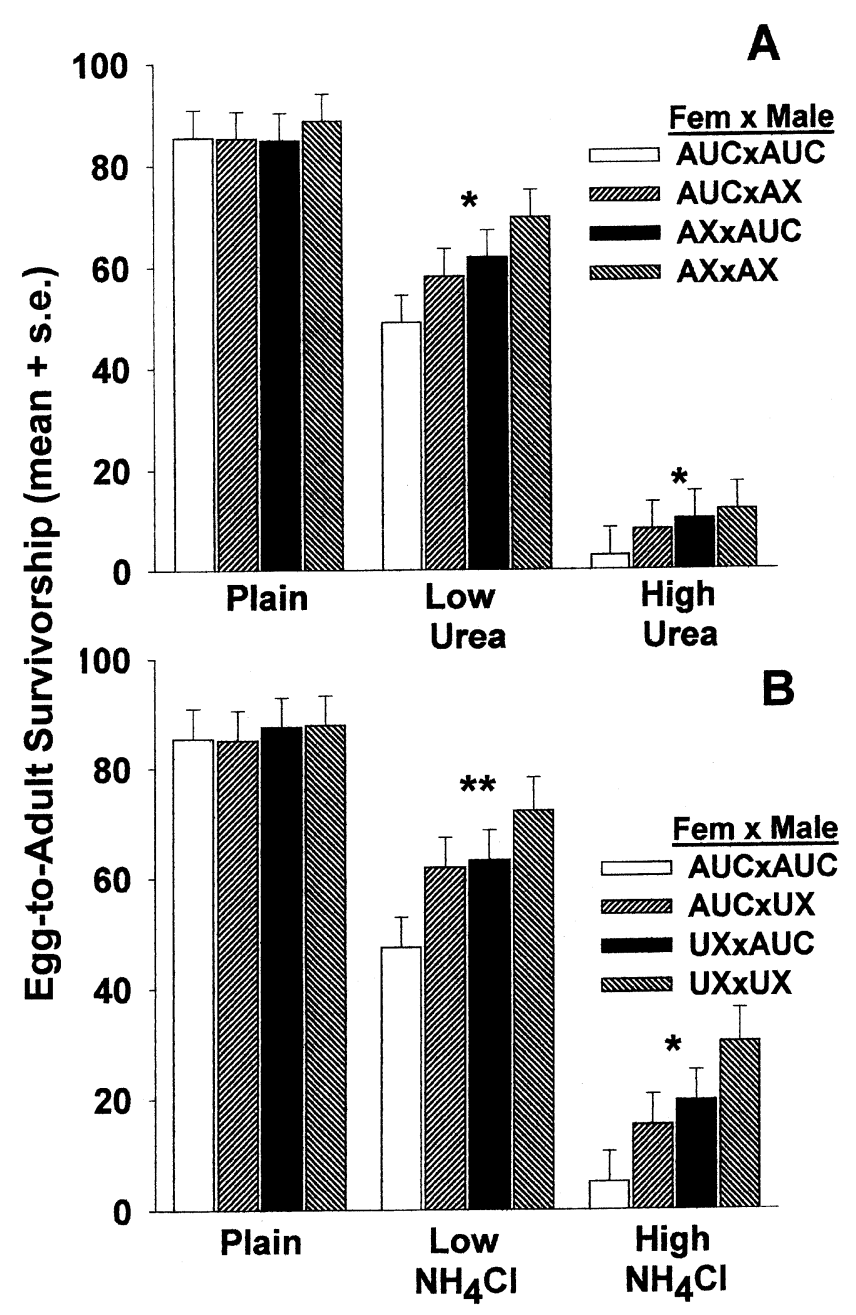

Fig. 3 Effect of larval exposure to urea and ammonia on eggto-adult survivorship and cross-tolerance in Drosophila melanogaster. (A) All AX, AUC, and their hybrid populations were measured on three types of food: plain banana food, banana food with $0.225 \mathrm{~mol}$ urea (Low), or banana food with 0.45 mol urea (High). (B) All UX, AUC, and their hybrid populations were measured on three types of food: plain banana food, banana food with $0.50 \mathrm{~mol} \mathrm{NH}_{4} \mathrm{Cl}$ (Low), or banana food with $1.00 \mathrm{~mol} \mathrm{NH}_{4} \mathrm{Cl}$ (High). The error bars are standard errors around the mean of the five replicate populations. Asterisks represent the significance of dominance controlling tolerance to the specific nitrogenous compound. $* P<0.05 ; * * P<0.01$.

the UX female $\times$ AX male, $\mathrm{X}$-linkage appears to affect survivorship. Similarly, the UX female $\times$ AX male populations showed a significantly higher viability on food containing low or high levels of urea, relative to the AX female $\times$ UX male, indicating that $X$-linkage may affect survivorship.

However, AX $\times$ AUC hybrids demonstrated significant dominance in egg-to-adult survivorship, when reared on either level of urea (Fig. 3A). Similarly, the
$\mathrm{UX} \times \mathrm{AUC}$ hybrids showed significant dominance in egg-to-adult survivorship, when reared on either level of ammonia (Fig. 3B).

\section{Developmental time}

Urea and ammonia tolerance. The ANOvA revealed that AX females (Fig. 4) and males (Fig. 5) developed fastest when reared as larvae on food containing either $0.50 \mathrm{~mol}$ or $1.00 \mathrm{~mol}$ of $\mathrm{NH}_{4} \mathrm{Cl}$, with the UX males and females demonstrating significant, although incomplete cross-tolerance for developmental time in the presence of $\mathrm{NH}_{4} \mathrm{Cl}$. UX females (Fig. 4) and males (Fig. 5) developed fastest when reared as larvae on food containing either low or high levels of urea, with the AX females and males demonstrating significant, although incomplete, cross-tolerance for developmental time in the presence of urea.

The ANOva for dominance revealed a significant population $\times \operatorname{sex} \times$ food interaction $\left(F_{8,32}=2.78, P<0.05\right)$, thus permitting multiple comparisons to be made. Reared on food containing either low or high urea levels, hybrid lines (AUC female $\times$ UX male and $\mathrm{UX}$ female $\times$ AUC male) displayed faster developmental times than their mid-parent values, indicating dominance. The ANOva for $\mathrm{X}$-linkage revealed a significant population $\times \operatorname{sex} \times$ food interaction $\left(F_{8,32}=3.08, P<0.02\right)$, thus permitting multiple comparisons to be made. Hybrid females and males from UX female $\times$ AUC male lines displayed faster developmental times relative to females and males from AUC female $\times$ UX male lines, on both $0.225 \mathrm{~mol}$ and 0.45 mol urea (Figs 4 and 5). This suggests that $X$-linkage is a factor controlling urea tolerance.

Reared on food containing either low $(0.5 \mathrm{~mol})$ or high $(1.0 \mathrm{~mol}) \quad \mathrm{NH}_{4} \mathrm{Cl}$ levels, hybrid lines (AUC female $\times$ AX male and AX female $\times$ AUC male) showed significant dominance for developmental times relative to their mid-parent values, indicating that dominance is involved in ammonia tolerance. Females and males from the AX female $\times$ AUC male lines eclosed sooner, relative to females and males from AUC female $\times$ AX male lines, on both low and high $\mathrm{NH}_{4} \mathrm{Cl}$ food. This suggests that $\mathrm{X}$-linkage and dominance control ammonia tolerance.

Cross-tolerance. Hybrid populations (AX female $\times \mathrm{UX}$ male and UX female $\times$ AX male) did not display faster developmental times relative to their mid-parent values, indicating that dominance does not significantly affect egg-to-adult developmental time for nitrogenous-compound-tolerant populations in novel environments (Fig. 6A, B). As the AX female $\times$ UX male lines showed a significantly faster developmental time on food containing low or high levels of $\mathrm{NH}_{4} \mathrm{Cl}(P<0.05$ for males on high urea food, $P<0.01$ for all others), relative to the 


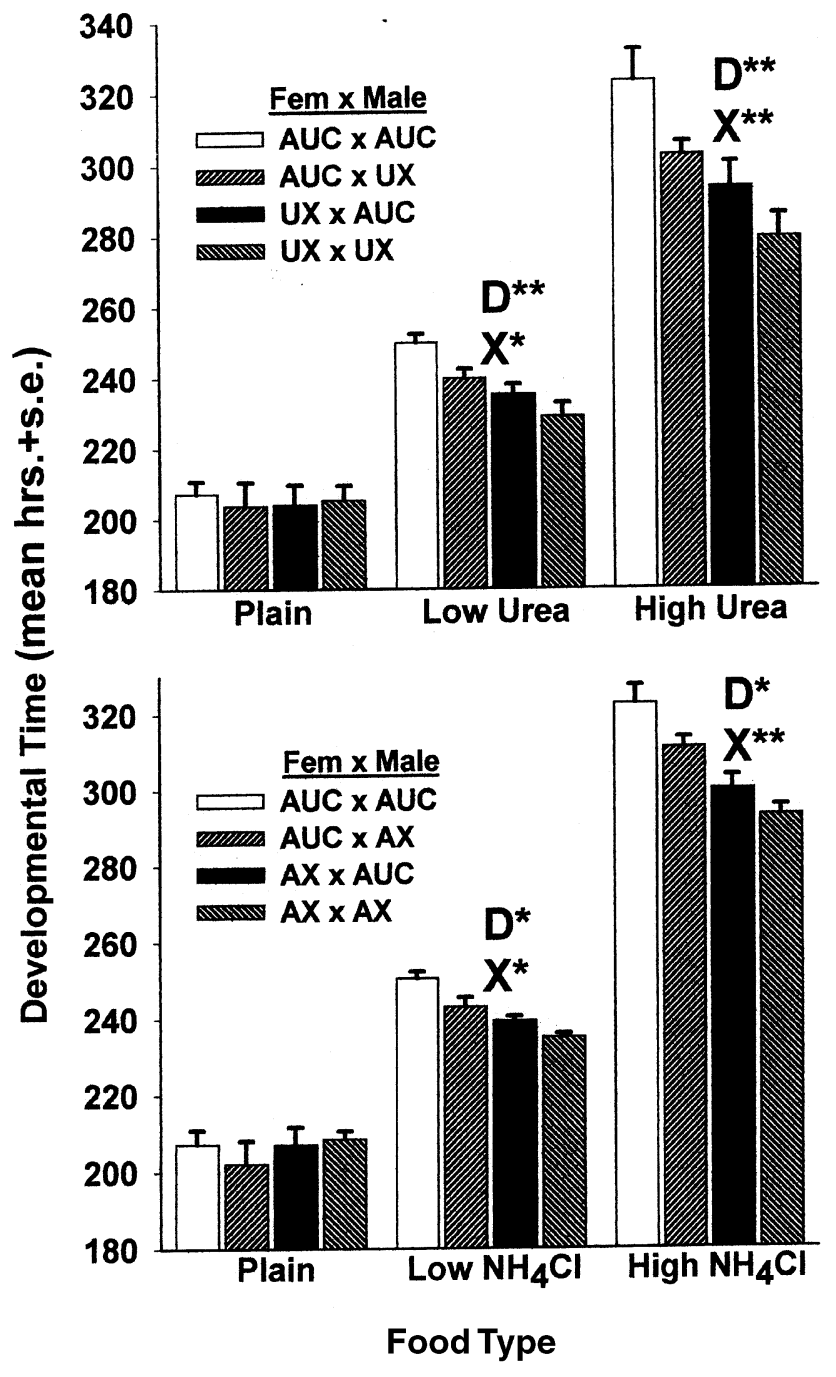

Fig. 4 Effect of larval exposure to urea and ammonia on female developmental time in Drosophila melanogaster. (A) Urea. All populations were measured on three types of food: plain banana food, banana food supplemented with $0.225 \mathrm{~mol}$ urea (Low), and banana food supplemented with 0.45 mol urea (High). (B) Ammonia. All populations were measured on three types of food: plain banana food, banana food supplemented with $0.50 \mathrm{~mol} \mathrm{NH}_{4} \mathrm{Cl}$ (Low), and banana food supplemented with $1.00 \mathrm{~mol} \mathrm{NH}_{4} \mathrm{Cl}$ (High). The error bars are standard errors around the mean of the five replicate populations. D represents the significance of dominance controlling tolerance to the specific nitrogenous compound. $\mathrm{X}$ represents the significance of $\mathrm{X}$-linkage controlling tolerance to the specific nitrogenous compound. X-linkage is demonstrated when a hybrid population, whose mother was from a stress-selected population (i.e. AX female $\times$ AUC male) shows significantly faster development than the reciprocal hybrid population, when both are reared on the same food medium. $* P<0.05 ; * * P<0.01$.

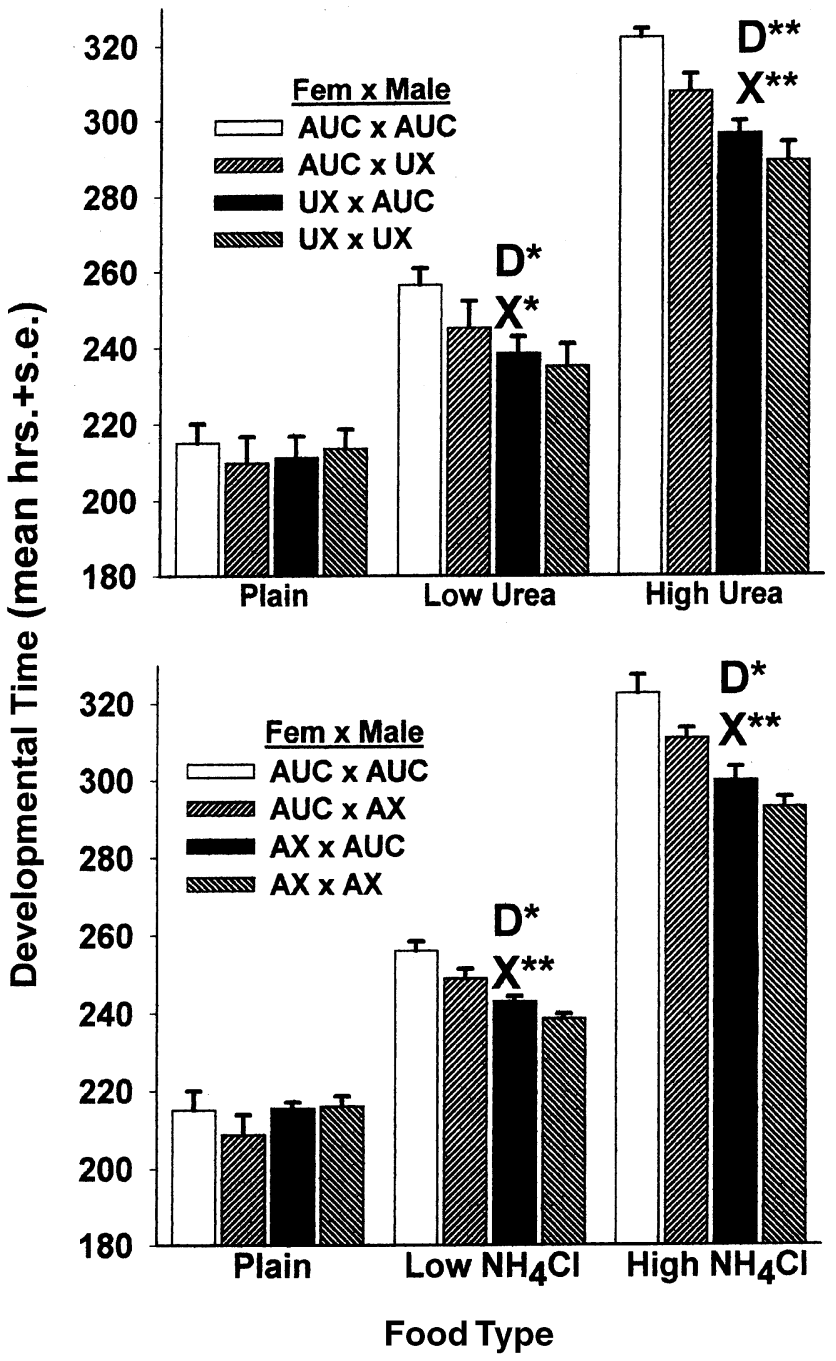

Fig. 5 Effect of larval exposure to urea and ammonia on male developmental time in Drosophila melanogaster. (A) Urea. All populations were measured on three types of food: plain banana food, banana food supplemented with $0.225 \mathrm{~mol}$ urea (Low), and banana food supplemented with 0.45 mol urea (High). (B) Ammonia. All populations were measured on three types of food: plain banana food, banana food supplemented with $0.50 \mathrm{~mol} \mathrm{NH}_{4} \mathrm{Cl}$ (Low), and banana food supplemented with $1.00 \mathrm{~mol} \mathrm{NH}_{4} \mathrm{Cl}$ (High). The error bars are standard errors around the mean of the five replicate populations. $\mathrm{D}$ represents the significance of dominance controlling tolerance to the specific nitrogenous compound, while $\mathrm{X}$ represents the significance of $\mathrm{X}$-linkage controlling tolerance to the specific nitrogenous compound. X-linkage is demonstrated when a hybrid population, whose mother was from a stressselected population (i.e. AX female $\times$ AUC male) shows significantly faster development than the reciprocal hybrid population, when both are reared on the same food medium. $* P<0.05 ; * * P<0.01$. 
UX female $\times$ AX male lines, $X$-linkage appears to affect larval development to a great degree. Similarly, UX female $\times \mathrm{AX}$ male lines demonstrated significantly faster developmental times on food containing low or high levels of urea $(P<0.05)$, relative to the $\mathrm{AX}$ female $\times$ UX male lines, indicating that X-linkage may affect egg-to-adult developmental time to a large extent.

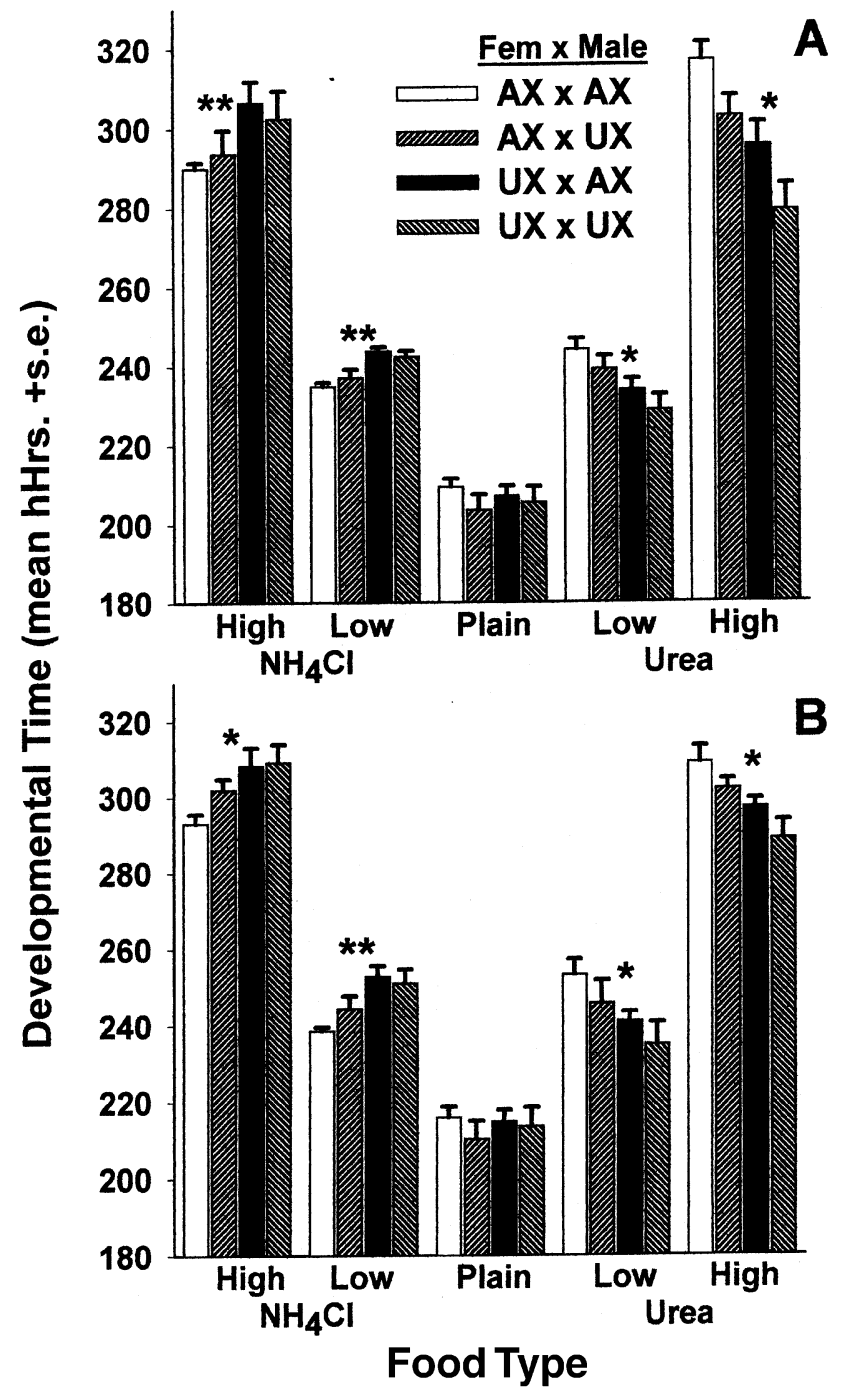

Fig. 6 Hybrids $(\mathrm{UX} \times \mathrm{AX}$ populations) in Drosophila melanogaster: developmental time and cross-tolerance. (A) Females. (B) Males. All AX, UX, and their hybrid populations were measured on five types of food: plain banana food, banana food supplemented with $0.225 \mathrm{~mol}$ urea (Low), banana food supplemented with 0.45 mol urea (High), banana food supplemented with $0.50 \mathrm{~mol} \mathrm{NH}_{4} \mathrm{Cl}$ (Low), and banana food supplemented with $1.00 \mathrm{~mol} \mathrm{NH}_{4} \mathrm{Cl}$ (High). The error bars are standard errors around the mean of the five replicate populations. The asterisks represent the significance of X-linkage controlling tolerance to the specific nitrogenous compound. $* P<0.05 ; * * P<0.01$.
However, AX $\times$ AUC hybrids demonstrated faster developmental times than their mid-parent values, when reared on either level of urea (Fig. 7A, B). Similarly, the $\mathrm{UX} \times \mathrm{AUC}$ hybrids demonstrated faster developmental times than their mid-parent values $(P<0.01)$, when reared on either level of ammonia (Fig. 7C, D). In both cases, dominance was detected without $\mathrm{X}$-linkage.

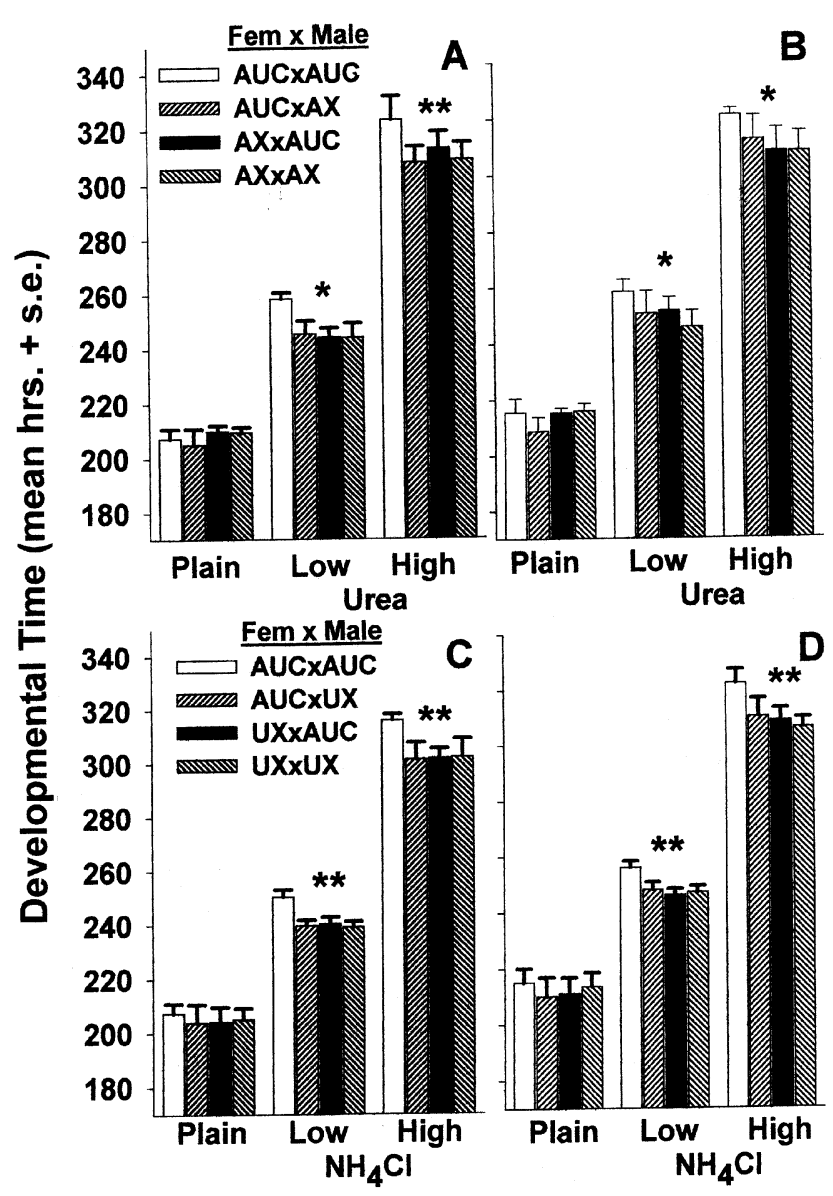

Fig. 7 Hybrids (stress selection populations $\times$ controls) in Drosophila melanogaster: developmental time and cross-tolerance. All AX, AUC, and their hybrid populations were measured on three food types: plain banana food, banana food supplemented with $0.225 \mathrm{~mol}$ urea (Low), banana food supplemented with 0.45 mol urea (High). (A) Females on urea. (B) Males on urea. All UX, AUC, and their hybrid populations were measured on three types of food: plain banana food, banana food supplemented with $0.50 \mathrm{~mol} \mathrm{NH}_{4} \mathrm{Cl}$ (Low), and banana food supplemented with $1.00 \mathrm{~mol} \mathrm{NH}_{4} \mathrm{Cl}$ (High).

(C) Females on ammonia. (D) Males on ammonia. The error bars are standard errors around the mean of the five replicate populations. The asterisks represent the significance of dominance controlling tolerance to the specific nitrogenous compound. Dominance is revealed by a greater proportion of both hybrid populations surviving to adulthood, relative to their mid-parents. ${ }^{*} P<0.05 ;{ }^{*} P<0.01$.

(C) The Genetics Society of Great Britain, Heredity, 86, 658-667. 


\section{Discussion}

As all populations had been removed from selection and reared in common environments for two generations prior to experimentation, differences among populations are genetic in origin. Additionally, as no experiments were performed on any $\mathrm{F}_{2}$ populations, it is impossible to determine whether observed X-linkage is influenced by cytoplasmic inheritance. As females inherit an X chromosome from each parent, observing differences between reciprocal hybrid females may indicate cytoplasmic inheritance influencing developmental time. In several cases (Fig. 4, Fig. 6A and Fig. 7A), females developed faster if they inherited their cytoplasmic material from a mother from a stress-selected population. However, as we did not perform $\mathrm{F}_{2}$ assays, we cannot eliminate cytoplasmic inheritance as a potential influence. Ideally, X-linkage would be more adequately measured through the comparison of $F_{1}$ males. However, we cannot be sure that an equal number of eggs of both genders were initially included in every vial at the start of the assays.

\section{Genetics of urea and ammonia tolerance}

Joshi et al. (1996) found that dominance and to some degree, X-linkage, were the primary genetic factors controlling developmental time and egg-to-adult survivorship, in a different series of independently created populations of Drosophila melanogaster, selected to tolerate high levels of larval urea. This observation is here confirmed in populations selected for urea tolerance. Hybrid populations show a superior ability to tolerate urea relative to the mid-parent values of the two parental populations, which suggests a strong dominance component for urea tolerance. As in Joshi et al. (1996), dominance was observed over two urea levels, indicating a stability of dominance. X-linkage is also observed for developmental time, as UX female $\times$ AUC male $F_{1} s$ developed to adulthood faster than AUC female $\times$ UX male $F_{1} s$.

A pattern of dominance and X-linkage is also observed in the AX population's evolved tolerance to $\mathrm{NH}_{4} \mathrm{Cl}$. Hybrid populations show superior ability to tolerate ammonia and display fast developmental times relative to the mid-parent values of the two parental populations. As with urea tolerance, dominance was seen over two ammonia levels, contrary to some results observed for other insects' tolerance (Georgihou \& Taylor, 1977; Taylor \& Georgihou, 1979), in which changes in insecticide level can affect whether resistance alleles are dominant or recessive.

\section{Genetics of cross-tolerance}

Borash et al. (2000a) demonstrated that populations selected to tolerate high larval levels of ammonia (AX) showed a higher egg-to-adult survivorship and faster developmental times in the presence of urea, relative to the unselected controls. Similarly, urea-selected (UX) populations showed a greater egg-to-adult survivorship and faster development times in the presence of ammonia, relative to the controls.

Dominance is not a factor controlling egg-to-adult survivorship and developmental time in the presence of a novel nitrogenous compound, for reciprocal crosses between the UX and AX populations. Hybrids, whose mother was obtained from a population selected to tolerate a specific nitrogenous compound, developed faster and, often, had greater egg-to-adult survivorship than the reciprocal hybrids, whose father was from a population selected to tolerate a specific nitrogenous compound. This suggests that substantial X-linkage or a cytoplasmic effect regulates some aspects of the crosstolerance phenomenon in the AX and UX populations. The dominance component of cross-tolerance was observed in the hybrid crosses between AUC and AX when reared on urea for egg-to-adult survivorship and developmental time, and with the AUC and UX hybrid crosses when reared on ammonia for both characteristics assayed.

One interpretation of these results is that there may exist two X-linked loci, one controlling urea tolerance and the other controlling ammonia tolerance. In this scenario, there are two alleles at each locus, with the alleles conferring tolerance to urea and to ammonia being dominant (Fig. 8). Additionally, there may exist one autosomal locus, which pleiotropically confers tolerance to both urea and ammonia. Again, the tolerance allele is dominant. The autosomal locus explains why males from, for example, AUC male $\times$ AX female hybrid populations, had greater egg-to-adult survivorship and faster developmental times than AUC populations, when reared on urea food. This may explain why we observed dominance for urea tolerance in the AUC $\times$ AX hybrids (Fig. 7A, B) and dominance for ammonia tolerance in the AUC $\times$ UX hybrids (Fig. 7C, D), but did not observe dominance (but only X-linkage) in the AX $\times$ UX hybrids (Fig. 6) on either food type. The pattern of dominance is clearly demonstrated for the UX $\times$ AUC hybrids reared on ammonia food and the AX $\times$ AUC hybrids reared on urea food. This, of course, assumes complete dominance and equality between the $\mathrm{X}$ and autosomal chromosome, which is unlikely, but it does represent the simplest explanation for the results 

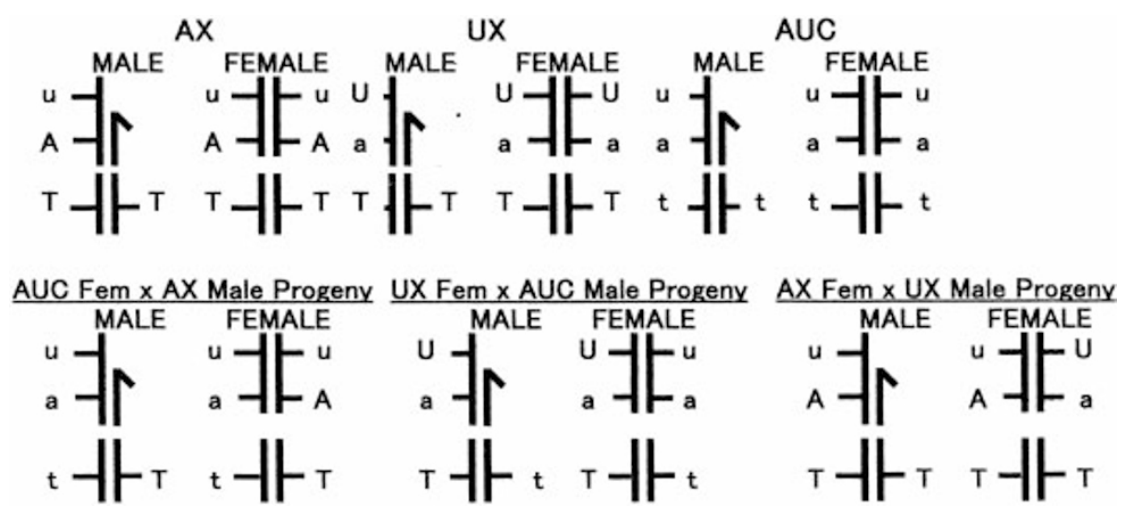

Fig. 8 A simple interpretation of the results in Drosophila melanogaster allowed the following model to be developed: there exists one autosomal loci that pleiotropically controls both urea and ammonia tolerance, and is dominant. At the same time, there are two $\mathrm{X}$-linked loci, one conferring urea tolerance and another conferring ammonia tolerance, both being dominant. The above graph depicts the pure-bred stock populations and the loci involved in tolerance to either urea, ammonia, or both. For the sake of brevity, we only include some of the hybrid crosses, and the resulting genotypes. In the above graph, the chromosome with both urea (U or u) and ammonia (A or a) tolerance loci represents the X-linked component of tolerance. The chromosome with the locus exerting pleiotropic control of both urea and ammonia tolerance ( $\mathrm{T}$ or $\mathrm{t}$ ), represents the autosomal chromosome. An upper case letter at a locus indicates the dominant allele (conferring resistance to either urea, ammonia, or in the case of the autosomal chromosome, both nitrogenous compounds), while the lower case letter represents the recessive allele. This scenario assumes that all dominant alleles contribute equivalent levels of tolerance to the organism.

observed. Our hypothesis neglects the possibility of modifier loci. Additionally, some codominance between the urea and ammonia tolerance alleles on an X-chromosome locus may exist. The interpretation above is also consistent with results seen in Joshi et al. (1996).

Selection for tolerance to extreme levels of nitrogenous compounds is clearly a component of densitydependent adaptation. In the case of Drosophila melanogaster, ammonia levels increase, among other changes in chemical composition (Hageman et al., 1990; Borash et al., 1998), as the culture ages. Here, in populations designed to study one aspect of densitydependent natural selection, we find that dominance and $\mathrm{X}$-linkage are the primary genetic mechanisms responsible for the observed tolerance of the UX populations to urea and the $\mathrm{AX}$ populations to ammonia.

\section{Acknowledgements}

We wish to thank L. D. Mueller for supplying us with the fruit flies. We thank A. Joshi and A. K. Chippindale for their comments and advice. Additional appreciation is given to K. Ohbayashi, F. Ishihama, and Y. Kurota for their assistance. This work is supported by grants from the Japanese Society for the Promotion of Sciences (JSPS P99127) and a Monbusho-Grant-in-Aid (11-99127) to DJB.

\section{References}

BORASH, D. J., GIBBS, A. G., JOSHI, A. AND MUELLER, L. D. 1998. A genetic polymorphism maintained by natural selection in a temporally varying environment. Am. Nat., 151, $148-156$.

BORASH, D. J., PIERCE, V. A., GIBBS, A. G. AND MUELleR, L. D. 2000a. Evolution of ammonia and urea tolerance in Drosophila melanogaster: resistance and cross-tolerance. J. Insect Physiol., 46, 763-769.

BORASH, D. J., TEOTONIO, H., ROSE, M. R. AND MUELLER, L. D. 2000b. Laboratory evolution of feeding rate and viability of Drosophila melanogaster. J. Evol. Biol., 13, 181-187.

BOTELlA, L. M., MOYA, A., GONZALEZ, M. C. AND MENSUA, J. L. 1985. Larval stop, delayed development and survival in overcrowded cultures of Drosophila melanogaster. Effect of urea and uric acid. J. Insect Physiol., 31, 179-185.

BURNETT, B., SEWELL, D. AND BOS, M. 1977. Genetic analysis of larval feeding behavior in Drosophila melanogaster II. Genet. Res., 30, 139-161.

FREEMAN, M. F. AND TUKEY, J. W. 1950. Transformation related to the angular and the square root. Ann. Math. Stat., 21, $607-611$.

GEORGIHOU, G. P. AND TAYLOR, C. E. 1977. Genetic and biological influences in the evolution of insecticide resistance. J. Econ. Entomol., 70, 319-323.

HAGEMAN, J. K., EISSES, T. H., JACOBS, P. J. M. AND SCHARLOO, W. 1990. Ethanol in Drosophila cultures is a selective factor. Evolution, 44, 447-454.

HOFFMANN, A. A. AND PARSONS, P. A. 1991. Evolutionary Genetics and Environmental Stress. Oxford University Press, New York. 
JOSHI, A. AND MUELLER, L. D. 1988. Evolution of higher feeding rate in Drosophila due to density dependent natural selection. Evolution, 42, 1090-1093.

JOSHI, A. AND MUELLER, L. D. 1996. Density-dependent natural selection in Drosophila. Trade offs between larval food acquisition and food utilization. Evol. Ecol., 10, 463-474.

JOSHI, A., KNIGHT, C. D. AND MUELLER, L. D. 1996. Genetics of larval urea tolerance in Drosophila melanogaster. Heredity, 77, 33-39.

MUELLER, L.D. 1995. Adaptation and density-dependent natural selection. In: Levine, L. (ed.) Genetics of Natural Populations: the Continuing Importance of Theodosius Dobzhansky, pp. 228-232. Columbia University Press, New York.
ROSE, M. R. 1984. Laboratory evolution of postponed senescence in Drosophila melanogaster. Evolution, 38, 1004-1010. SHIOTSUGU, J., LEROI, A. M., YASHIRO, H., ROSE, M. R. ET AL. 1997. The symmetry of correlated selection responses in adaptive evolution: an experimental study using Drosophila. Evolution, 51, 163-172.

SOMERO, G. N. AND YANCEY, P. H. 1997. Osmolytes and cellvolume regulation: physiological and evolutionary principles. In: Hoffman, F. F. and Jamieson, J. D. (eds) Handbook of Physiology. Oxford University Press, Oxford, UK.

TAYLOR, C. E. AND GEORGIHOU, G. P. 1979. Suppression of insecticide resistance by alteration of gene dominance and migration. J. Econ. Entomol., 72, 105-109. 